\title{
INVERSE METHOD FOR A ONE-STAGE SPUR GEAR DIAGNOSIS
}

\author{
Ali Akrout, Dhouha Tounsi, Mohamed Taktak, Mohamed Slim Abbes, \\ MOHAMED HADDAR \\ Mechanics, Modelling and Production Research Laboratory (LA2MP), National School of Engineers of Sfax, \\ University of Sfax, Sfax, Tunisia; e-mail: mohamed.taktak@fss.rnu.tn; mohamed.taktak.tn@gmail.com
}

\begin{abstract}
In this paper, a source separation approach based on the Blind Source Separation (BSS) is presented. In fact, the Independent Component Analysis (ICA), which is the main technique of BSS, consists in extracting different source signals from several observed mixtures. This inverse method is very useful in many fields such as telecommunication, signal processing and biomedicine. It is also very attractive for diagnosis of mechanical systems such as rotating machines. Generally, dynamic responses of a given mechanical system (displacements, accelerations and speeds) measured through sensors are used as inputs for the identification of internal defaults. In this study, the ICA concept is applied to the diagnosis of a one-stage gear mechanism in which two types of defects (the eccentricity error and the localized tooth defect)are introduced. The finite element method allows determination of the signals corresponding to the acceleration in some locations of the system, and those signals may be used also in the ICA algorithm. Hence, the vibratory signatures of each defect can be identified by the ICA concept. Thus, a good agreement is obtained by comparing the expected default signatures to those achieved by the developed inverse method.
\end{abstract}

Keywords: Independent Component Analysis (ICA), source identification, gear mechanism, geometrical defects

\section{Introduction}

Diagnosis, without disassembling different parts of a given machine, represents the main purpose to be achieved in the defect detection practice. This procedure allows the industry to save time and money. That is why many approaches found in the literature can be considered as tools to realize non destructive diagnosis of machines. Usually, any internal default or external force which excites mechanical systems can be identified by frequency analysis. That allows the determination of the internal default excitation frequencies or the external excitation forces. However, the knowledge of the different excitation frequencies existing in the spectrum of the system vibratory signal is insufficient to determine the type of defaults, its amplitude and its position in the system. To surpass these limitations, various methods have been developed in order to solve the source separation problem leading to different algorithms which can be used for modal analysis of structures and dynamic systems (Antoni et al., 2004; Poncelet et al., 2007; Kerschen et al., 2007; Abbès et al., 2011; Akrout et al., 2012a,b). One of these techniques is the Blind Source Separation (BSS) which was presented by many researchers (Antoni and Braum, 2005) and (Antoni, 2005). It consists in separating an independent linear mixture source signal without any information about the sources by using only the measured vibratory response of the system. The separation procedure is based on the statistical independency of sources.

In the last few years, an increasing number of works using the ICA concept for detecting defects in mechanical systems have been found. Many researchers have developed different algorithms based on the ICA technique for the diagnosis of rotating machines (Servière and Fabry, 
2004) and (Taktak et al., 2012), bearings (Shalvi and Weinstein, 1990) and structures (Zang and Friswel, 2004), (Akrout et al., 2012a,b).

In this paper, the ICA method is applied to diagnosis a one-stage spur gear system. The spur gear represents a device that ensures transmission of movement in various mechanical systems. In a gear mechanism, there appear frequently many internal defaults which generate disturbances in the dynamic responses. By using the ICA concept, both the amplitude and the frequency are used to localize the defaults.

After presentation of the separation method including the definition of the ICA in Section 2, Section 3 presents the separation hypothesis of the ICA concept and the associated different steps. Section 4 presents the results and the discussion of the application of this technique to a one-stage spur gear to identify its internal defaults.

It is also worth mentioning that in our previous work (Taktak et al., 2012), the observed signals (accelerations) are determined on two nodes of a discretized crankcase flexible plate. However, in this article, the vibratory responses are calculated in the pinion and the gear centre, respectively.

\section{Inverse method for dynamic problem: the Independent Component Analysis} (ICA)

\subsection{ICA definition}

The ICA method was defined by Comon (1990) as a statistical technique that aims at decomposing a random signal $\mathbf{X}$ (measured signal) into a linear combination of independent signals (source signals). Then, the ICA problem is formulated as follows

$$
\mathbf{X}=\mathbf{A S}
$$

where $\mathbf{X}$ is a column vector of $M$ output observations, which represents an instantaneous linear mixture of source signals $\mathbf{S}$ defined by a column vector of $N$ sources. $\mathbf{A}$ is a $M \times N$ mixing matrix. The equation of the ICA concept is described as follows (Zang et al., 2004; Abbès et al., 2011; Akrout et al., 2012)

$$
X_{j}(t)=\sum_{i=1}^{N} a_{j i} S_{i}(t) \quad j=1, \ldots, M
$$

\subsection{ICA indeterminacies}

In spite of its advantages in separating source signals, two ambiguities are inherent to the ICA method: it aims at identification of both source signals and the mixing matrix with certain indeterminacies that include arbitrary scaling and permutation. The mixture model can be expressed as follows (Antoni, 2005; Tong et al., 1991)

$$
\mathbf{X}=\mathbf{A} \mathbf{P}^{-1} \mathbf{P S}
$$

where $\mathbf{P}$ is the permutation matrix.

In the same way, the observations can be rewritten as follows

$$
\mathbf{X}=\mathbf{A D}^{-1} \mathbf{D S}
$$

D is a diagonal matrix. So any scalar multiplier to one of the source $S_{i}$ could be avoided by dividing the corresponding column of the matrix $\mathbf{A}$. 
These two indeterminacies imply that it is impossible to obtain a unique determination of the original sources. So the separating matrix $\mathbf{B}$ is estimated by the product $\mathbf{B A}$ which is equal to the diagonal matrix $\mathbf{D}$ up to the permutation matrix $\mathbf{P}$

$$
\mathbf{B A}=\mathbf{D P}
$$

In order to reduce the shape indeterminacy in the model and save the waveform of the original signals, a constraint of unit variance is imposed on the sources $\mathbf{S}$ to eliminate the scale factor (Antoni, 2005; Tong et al., 1991)

$$
E\left[\mathbf{S S}^{+}\right]=\mathbf{I}
$$

where $(+)$ denotes the conjugate, and $\mathbf{I}$ is the $N \times N$ identity matrix.

The ICA concept assumes that the sources Si must be statically independent uncorrelated and have a non-Gaussian distribution. Then, to perform the estimated source signals task and the mixing matrix $\mathbf{A}$, the ICA requires some separation assumptions which are presented in the following section.

\subsection{Separation assumptions}

\subsubsection{Statistical independency of the sources}

The separation can be achieved only when all the components of the separating system are mutually and statically independent. The components of a random vector $\mathbf{X}$ are mutually independent if and only if the probability density of the vector $\mathbf{X}$ is equal to the marginal probability density (Akrout et al., 2012a,b; Hyvärinen and Oja, 2000)

$$
P_{x}(x)=\prod_{i=1}^{N} P_{x_{i}}\left(x_{i}\right)
$$

Many formulations are used to assure the statistical independence of the sources such as the mutual information which is based on the Kulback divergence (Comon, 1990)and the likelihood estimation (Geata and Lacoume, 1990).

\subsubsection{Uncorrelated sources}

The statistical independence of the sources imposes that the sources must be uncorrelated. So, two variables $Y_{1}$ and $Y_{2}$ are uncorrelated if and only if their covariance is equal to zero (Akrout et al., 2012a,b; Comon, 1990)

$$
E\left\{Y_{1} Y_{2}\right\}-E\left\{Y_{1}\right\} E\left\{Y_{2}\right\}=0
$$

\subsection{Non-Gaussianity distribution of the sources}

Another very useful hypothesis in the source separation is the non-Gaussianity of the sources (Akrout et al., 2012a,b; Comon, 1990). This criterion is especially essential in the case of stationary white sources since the non-Gaussianity of the sources is needed to achieve the separation.

The Gaussianity of a signal is defined as the difference between the distributions of the signal and the Gaussian signal with the same power. Instantaneous linear mixture sources can be separated by maximizing the non-Gaussianity of the output signal, which is obtained by a linear combination of the observations. 
The non-Gaussianity can be evaluated by the kurtosis or the fourth order cumulate. The Kurtosis of a variable $y$ is expressed as follows (Comon, 1990)

$$
\operatorname{Kurt}(y)=E\left\{y^{4}\right\}-3\left(E\left\{y^{2}\right\}\right)^{2}
$$

Another measure of non-Gaussianity which can be used is the negentropy theory which is based on the theoretical quantity of the differential entropy (Wiggins, 1978). The differential entropy of a variable $y$ with a density $f(y)$ can be defined as follows (Hyvärinen and Oja, 2000)

$$
H(y)=-\int f(y) \log (f(y)) d y
$$

So, the negentropy which is equal to zero for the Gaussian variable can be written as follows (Hyvärinen and Oja, 2000)

$$
J(y)=H\left(y_{\text {gauss }}\right)-H(y)
$$

where $y_{\text {gauss }}$ is a random variable which has the same covariance matrix as $y$. So, the negentropy is always non negative and it is equal to zero in the case of Gaussian variable.

\section{ICA steps}

\subsection{Pre-processing for ICA}

Before applying the ICA algorithm and to guarantee the uncorrolatedness of the sources, it is useful to do some pre-processing. These pre-processings make the ICA estimation easy. These pre-processings are presented in the following sections.

\subsubsection{Centring}

The first pre-treatment is the centring. Each observed vector $\mathbf{X}$ must be centred, i.e., subtracts its mean vector $m=E\{\mathbf{X}\}$; so, $\mathbf{X}$ will be the zero mean variable which implies that $\{S\}$ is the zero mean as well (Hyvärinen and Oja, 2000).

\subsubsection{Whitening}

The second pre-treatment used in ICA is whitening of the observations $\mathbf{X}$. This means that before applying the ICA algorithm, we transform the vector $\mathbf{X}$ to a white vector $\widetilde{\mathbf{X}}$ which has uncorrelated components, and the variance of its components is equal to unity and (Thirion-Moreau, Moreau, 2000)

$$
\widetilde{\mathbf{X}}=\mathbf{W X}
$$

$\mathbf{W}$ is the whitening matrix, it is determined by the eigenvalues decomposition of the covariance matrix which is defined by

$$
R_{x}(0)=E\left\{\mathbf{X} \mathbf{X}^{+}\right\}=\mathbf{A} \mathbf{A}^{+}=\mathbf{U D U}^{\mathrm{T}}
$$

where $\mathbf{U}$ is the eigenvector orthogonal matrix of $E\left\{\mathbf{X X}^{\mathrm{T}}\right\}$ and $\mathbf{D}$ is the diagonal matrix of its eigenvalues.

So,we can write

$$
E\left\{(\mathbf{W X})(\mathbf{W X})^{+}\right\}=\mathbf{W} \mathbf{W}^{+} E\left\{\mathbf{X X}^{+}\right\}=\mathbf{I}
$$


Then, the whitening matrix can be defined as follows

$$
\mathbf{W}=\mathbf{D}^{-1 / 2} \mathbf{U}^{\mathrm{T}}
$$

This transformation allows definition of a new matrix $\widetilde{\mathbf{A}}$

$$
\widetilde{\mathbf{X}}=\mathbf{D}^{-1 / 2} \mathbf{U}^{\mathrm{T}} \mathbf{A} \mathbf{S}=\widetilde{\mathbf{A}} \mathbf{S}
$$

$\widetilde{\mathbf{A}}$ is an orthogonal mixing matrix, then we can write

$$
E\left\{\widetilde{\mathbf{X}} \widetilde{\mathbf{X}}^{\mathrm{T}}\right\}=\widetilde{\mathbf{A}} E\left\{\mathbf{S S}^{\mathrm{T}}\right\} \widetilde{\mathbf{A}}^{\mathrm{T}}=\widetilde{\mathbf{A}} \widetilde{\mathbf{A}}^{\mathrm{T}}=\mathbf{I}
$$

So, the whitening of the observations contributes to reduction of the number of parameters to be estimated. Instead of estimating $n^{2}$ parameters, only the elements of the new orthogonal matrix $\widetilde{\mathbf{A}}$ are considered. We noticed that an orthogonal matrix has $n(n-1) / 2$ degrees of freedom (Hyvärinen and Oja, 2000).

\subsection{ICA algorithm}

\subsubsection{Kurtosis maximization}

In the literature, many contrast functions have been proposed. Among them the Kurtosis defined by the normalized fourth order marginal cumulate is the commonly used statistical quantity in the ICA concept (Comon, 1994). The kurtosis was firstly used in blind separation of seismic signals and digital communication (Shalvi and Weinstein, 1990; Wiggins, 1978).

The separation of sources using ICA is based on the determination of each column of the separating matrix $\mathbf{W}$. Then it determines the source related to this column. The extracting vector of the mixing matrix can be defined as follows (Akrout et al., 2012a,b; Zarzoso and Comon, 2010)

$$
\mathbf{Y}=\mathbf{W}^{\mathrm{H}} \mathbf{X}
$$

where $\mathbf{Y}$ is the estimated source and $\mathbf{W}$ is the separating matrix, $(\cdot)^{\mathrm{H}}$ denotes the conjugate transpose operator. W must maximize the Kurtosis in order to guarantee the non-Gaussianity distribution. This Kurtosis is defined as follows (Akrout et al., 2012a,b; Zarzoso and Comon, 2010)

$$
K(\omega)=\frac{E\left\{|y|^{4}\right\}-2 E^{2}\left\{|y|^{2}\right\}-\left|E\left\{y^{2}\right\}\right|^{2}}{E^{2}\left\{|y|^{2}\right\}}
$$

In order to maximize this function, an exact line search of the absolute Kurtosis contrast is performed, which is defined as (Zarzoso and Comon, 2010)

$$
\mu_{\text {opt }}=\arg (K(w+\mu g))
$$

where $g$ is the search direction defined as the gradient $g=\nabla_{w}(K(\omega))$. The exact line search is determined after determination of roots of a fourth degrees polynomial, so the roots leading to the absolute maximum of the contrast along the search direction are chosen.

After the determination of the column of the separating matrix, a deflation approach is considered in order to extract the source corresponding to the determined column.

\subsubsection{Deflation approach}

The deflation approach allows escaping from a multiple inputs/multiple outputs system into a system with a single input/multiple outputs. The deflation approach is used to extract successively the sources. This ensures that each source is chosen once with multiplying the exact factor. After the determination of the column vector of the separation matrix $\mathbf{W}$ by maximizing the kurtosis contrast, the deflation approach is used in order to determine the estimated source. 


\section{Diagnosis of a one-stage gear mechanism}

The purpose of this part is to provide an illustration of the capability of the ICA algorithm to separate and extract signals from a given mechanical system which is a one-stage spur gear mechanism. The ICA concept is applied to this system and the source identification results are presented and discussed.

\subsection{System presentation}

The studied system is a one-stage spur gear mechanism. It can be divided into two blocks as presented in Fig. 1a. The first block is composed by the input shaft (1) and a pinion. The second block is constituted by the gear and the output shaft (2). Both the pinion and the gear are supposed to be rigid. The shafts, which are assumed to be flexible, are discretized by two node beam finite elements (Fig. 1b) with 6 degrees of freedom per node.
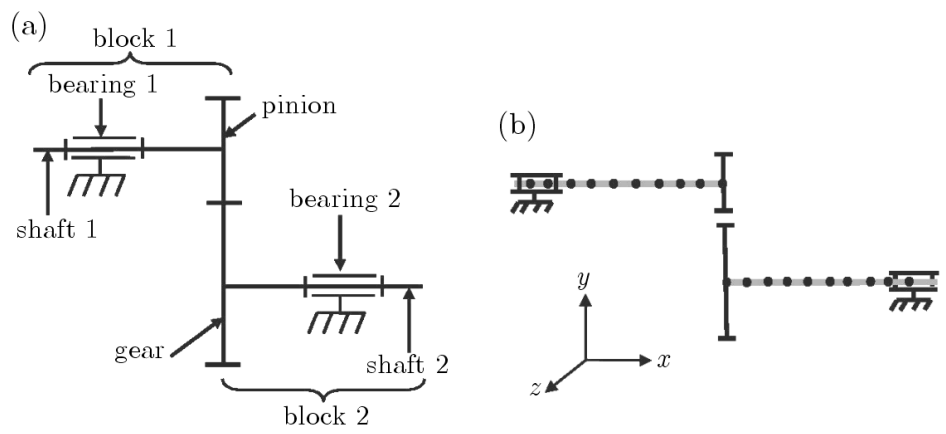

Fig. 1. Studied case: (a) model of a one-stage spur gear, (b) discretized single spur gear

The shafts are supported by bearings each modelled by two linear springs. The gear mesh stiffness is modelled by a linear spring acting on the line of action of the meshing teeth.

The expression of the displacement on the line of action is expressed as (Chaari et al., 2008)

$$
\delta(t)=\left(z_{1}-z_{2}\right) \sin \gamma+\left(y_{1}-y_{2}\right) \cos \gamma+\theta_{x p} r_{b p}+\theta_{x g} r_{b g}
$$

where $z_{i}$ and $y_{i}(i=1,2)$ are respectively the centre displacements of the pinion and gear. $\theta_{x p}$ and $\theta_{x g}$ are angular rotations of the pinion and the gear centre, respectively. $r_{b p}$ and $r_{b g}$ are the basis radius of the pinion and the gear, respectively. $\gamma$ is the pressure angle.

The equation of motion of the system is defined as (Chaari et al., 2008)

$$
\mathbf{M} \ddot{\mathbf{q}}+\mathbf{C} \dot{\mathbf{q}}+\mathbf{K}(t) \mathbf{q}=\mathbf{F}_{\text {ext }}
$$

where $\mathbf{M}, \mathbf{C}$ and $\mathbf{K}(t)$ are respectively the global mass, damping and stiffness matrices of the whole system. $\mathbf{q}$ is the vector of degrees of freedom, $\mathbf{F}_{\text {ext }}$ is the external applied forces vector.

The mass matrix $\mathbf{M}$ is a diagonal matrix which can be written as follows (Chaari et al., 2008)

$$
\mathbf{M}=\left[\begin{array}{cccc}
\mathbf{M}_{1} & \mathbf{0} & \mathbf{0} & \mathbf{0} \\
\mathbf{0} & \mathbf{M}_{p} & \mathbf{0} & \mathbf{0} \\
\mathbf{0} & \mathbf{0} & \mathbf{M}_{g} & \mathbf{0} \\
\mathbf{0} & \mathbf{0} & \mathbf{0} & \mathbf{M}_{2}
\end{array}\right]
$$

$\mathbf{M}_{i}(i=1,2)$ is the mass matrix of the $i$ th shaft; $\mathbf{M}_{p}$ and $\mathbf{M}_{g}$ are respectively the mass matrices of the pinion and the gear (Chaari et al., 2008).

$\mathbf{K}(t)$ includes the shafts stiffness, bearing stiffness and the time varying gear mesh stiffness matrix.The stiffness matrix of the shafts is determined by the FE model presented in Fig. 1b. 
Then, the matrix $\mathbf{K}(t)$ can be divided into a mean stiffness matrix $\overline{\mathbf{K}}$ and a fluctuating one $\mathbf{K}_{G S}(t)$ (Chaari et al., 2008)

$$
\begin{aligned}
& \mathbf{K}(t)=\overline{\mathbf{K}}+\mathbf{K}_{G S}(t) \\
& \overline{\mathbf{K}}=\left[\begin{array}{cccc}
\mathbf{K}_{\text {shaft'1 }} & \mathbf{K}_{C 1} & \mathbf{0} & \mathbf{0} \\
\mathbf{K}_{C 1}^{\mathrm{T}} & \mathbf{K}_{\text {bearing }} 1^{\prime} & \mathbf{0} & \mathbf{0} \\
\mathbf{0} & \mathbf{0} & \mathbf{K}_{\text {bearing }} 2^{\prime} & \mathbf{K}_{C 2} \\
\mathbf{0} & \mathbf{0} & \mathbf{K}_{C 2}^{\mathrm{T}} & \mathbf{K}_{\text {shaft }} 2^{\prime}
\end{array}\right]
\end{aligned}
$$

$\mathbf{K}_{\text {shaft } i^{\prime}}$ and $\mathbf{K}_{\text {bearing }} i^{\prime}(i=1,2)$ are the stiffness matrices of the $i$ th shaft. $\mathbf{K}_{C 1}$ and $\mathbf{K}_{C 2}$ are the coupling matrices between the bearings and shafts. The bearing stiffness matrices contain the translation stiffness along with the $z$ and $y$ directions (Chaari et al., 2008) $\left(K_{y 1}=K_{z 1}=\right.$ $\left.K_{y 2}=K_{z 2}=10^{8} \mathrm{~N} / \mathrm{m}\right)$.

$\mathbf{K}_{G S}(t)$ is the fluctuation stiffness matrix defined as follows (Chaari et al., 2008)

$$
\mathbf{K}_{G S}(t)=\left[\begin{array}{cccccccccccc}
0 & 0 & 0 & 0 & 0 & 0 & 0 & 0 & 0 & 0 & 0 & 0 \\
0 & s_{4} & s_{5} & s_{6} & 0 & 0 & 0 & -s_{4} & -s_{5} & s_{8} & 0 & 0 \\
0 & s_{5} & s_{3} & s_{7} & 0 & 0 & 0 & -s_{5} & s_{3} & s_{9} & 0 & 0 \\
0 & s_{6} & s_{7} & s_{10} & 0 & 0 & 0 & -s_{6} & s_{7} & s_{12} & 0 & 0 \\
0 & 0 & 0 & 0 & 0 & 0 & 0 & 0 & 0 & 0 & 0 & 0 \\
0 & 0 & 0 & 0 & 0 & 0 & 0 & 0 & 0 & 0 & 0 & 0 \\
0 & 0 & 0 & 0 & 0 & 0 & 0 & 0 & 0 & 0 & 0 & 0 \\
0 & -s_{4} & -s_{5} & -s_{6} & 0 & 0 & 0 & s_{4} & s_{5} & -s_{8} & 0 & 0 \\
0 & -s_{5} & -s_{3} & -s_{7} & 0 & 0 & 0 & s_{5} & s_{3} & -s_{9} & 0 & 0 \\
0 & s_{8} & s_{9} & s_{12} & 0 & 0 & 0 & -s_{8} & -s_{9} & s_{11} & 0 & 0 \\
0 & 0 & 0 & 0 & 0 & 0 & 0 & 0 & 0 & 0 & 0 & 0 \\
0 & 0 & 0 & 0 & 0 & 0 & 0 & 0 & 0 & 0 & 0 & 0
\end{array}\right] K_{e}(t)
$$

where $K_{e}(t)$ is the time varying gear mesh stiffness.

The terms $s_{i}(i=1, \ldots, 12)$ are given in Table 1 .

Table 1. Coefficients $s_{i}$

\begin{tabular}{|c|c|c|c|}
\hline$s_{1}$ & $\sin \gamma$ & $s_{7}$ & $r_{b p} \sin \gamma$ \\
\hline$s_{2}$ & $\cos \gamma$ & $s_{8}$ & $r_{b g} \cos \gamma$ \\
\hline$s_{3}$ & $\sin ^{2} \gamma$ & $s_{9}$ & $r_{b g} \sin \gamma$ \\
\hline$s_{4}$ & $\cos ^{2} \gamma$ & $s_{10}$ & $r_{b p}^{2}$ \\
\hline$s_{5}$ & $\sin \gamma \cos \gamma$ & $s_{11}$ & $r_{b g}^{2}$ \\
\hline$s_{6}$ & $r_{b p} \cos \gamma$ & $s_{12}$ & $r_{b g} r_{b p}$ \\
\hline
\end{tabular}

The damping matrix of the system is determined from a combination between the mass matrix of the system and the mean stiffness matrix

$$
\mathbf{C}=\alpha \mathbf{M}+\beta \overline{\mathbf{K}}
$$

where $\alpha$ and $\beta$ are two constants which influence the stability of the system. The properties of the studied system are presented in Tables 2 and 3 .

\subsection{Geometrical and teeth defect models}

In order to disturb the dynamic response of the studied system, some defects have been introduced into the spur gear. The vibration sources (internal and external excitations) of the dynamic transmission are multiple. However, the internal sources have considerable effects on 
Table 2. Parameters of the spur gear (Hidaka et al., 1979)

\begin{tabular}{|l|c|c|}
\cline { 2 - 3 } \multicolumn{1}{c|}{} & Pinion & Gear \\
\hline \hline Teeth numbers & 20 & 40 \\
\hline Mass $[\mathrm{kg}]$ & 0.6659 & 2.663 \\
\hline Inertia Moment $\left[\mathrm{kg} \mathrm{m}^{2}\right]$ & $2.996 \cdot 10^{-4}$ & 0.0048 \\
\hline Base diameter circle $[\mathrm{m}]$ & 0.0564 & 0.1128 \\
\hline Rotation speed [rpm] & 1500 & 750 \\
\hline Torque $[\mathrm{Nm}]$ & 10 & -20 \\
\hline Module $[\mathrm{mm}]$ & 3 & \\
\hline Pressure angle $[\mathrm{deg}]$ & 20 & \\
\hline Gear mesh frequency $[\mathrm{Hz}]$ & 500 & \\
\hline
\end{tabular}

Table 3. Parameters of the input and output shafts (Hidaka et al., 1979)

\begin{tabular}{|c|c|}
\hline Shaft characteristics & value \\
\hline Material & steel \\
\hline Young's modulus $[\mathrm{MPa}]$ & $2 \cdot 10^{5}$ \\
\hline Length $[\mathrm{m}]$ & 0.45 \\
\hline Section $\left[\mathrm{m}^{2}\right]$ & $3.84 \cdot 10^{-3}$ \\
\hline Poisson's coefficient & 0.29 \\
\hline Density $\left[\mathrm{kg} / \mathrm{m}^{3}\right]$ & 7858 \\
\hline
\end{tabular}

the dynamic response of the system. As examples, of internal excitation sources, we can mention assembly defects which are characterized by an excitation frequency which corresponds to the rotation frequency of the shafts as well as geometric deviation which is characterized by an excitation frequency corresponding to the gear mesh rotation frequency.

The geometric deviation comes mainly from manufacturing defects; they are duplicated typically at the gear mesh frequency. We can distinguish the profile error which presents the difference between the actual profile and the theoretical profile of the teeth. According to Munro (1967), the profile error which is duplicated on each tooth, induces a transmission error marked at the gear mesh frequency and its harmonics.

The eccentricity error represents the difference between the real and theoretical location of the axes of the gear polar moment of inertia. Munro (1967) shows that the eccentricity of the wheel is characterized by the presence of a main peak at the rotation frequency in the spectrum of the static transmission.

Furthermore, teeth defects, such as spalling, breakage and crack, can affect considerably the dynamic behaviour of the gear transmission. In that case, several dynamic models have been developed in order to achieve some diagnosis solutions (Munro, 1967; Chaari et al., 2009).

The eccentricity error and the localized tooth defect have the following expressions (Hidaka et al., 1979; Wajag and Kahraman, 2002)

$$
F_{d e f_{1}}=K_{e}(t) e_{c} \sin \left(2 \pi f_{r} t\right) \quad F_{d e f_{2}}=K_{e}(t) e_{c} \sin \left(2 \pi f_{e n} t\right)
$$

where $f_{r}$ is the rotation frequency of the shaft, $e_{c}$ is the excitation amplitude, $f_{e n}$ is the gear mesh frequency. Therefore, the equation of motion of the spur gear transmission can be expressed as follows

$$
\mathbf{M}, \ddot{\mathbf{q}}+\mathbf{C} \dot{\mathbf{q}}+\mathbf{K}(t) \mathbf{q}=\mathbf{F}-\mathbf{F}_{d e f 1}-\mathbf{F}_{d e f 2}
$$

Equation (4.10) is solved using the New mark method. Then, vibratory responses of the system are determined and used to reconstruct the forces related to different system failures. 


\subsection{Numerical simulation}

As mentioned above, the first step in the simulation process is the introduction of both eccentricity and tooth defaults. The waveforms of the two internal defects introduced in the gear transmission are presented in Figs. 2 and 3. The first one (Fig. 2) corresponds to the temporary evolution of the eccentricity error. The second one (Fig. 3) corresponds to the time evolution of the tooth defect.

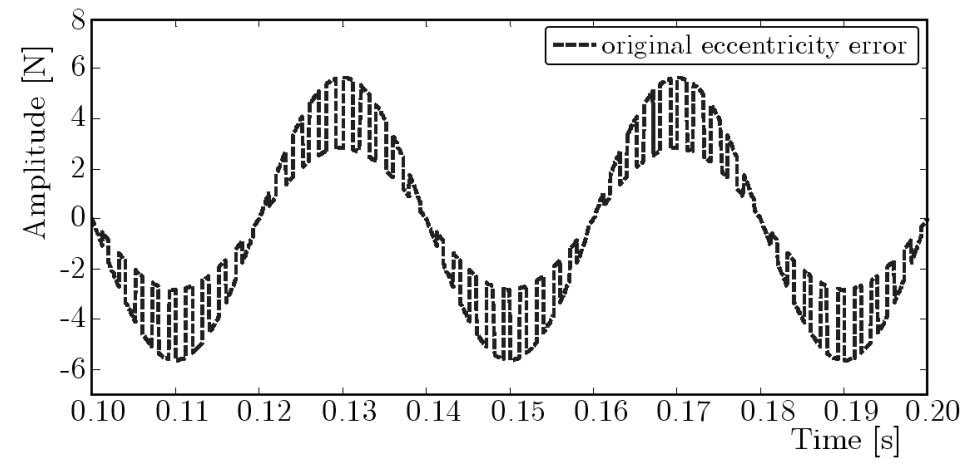

Fig. 2. Time-evolution of the eccentricity error

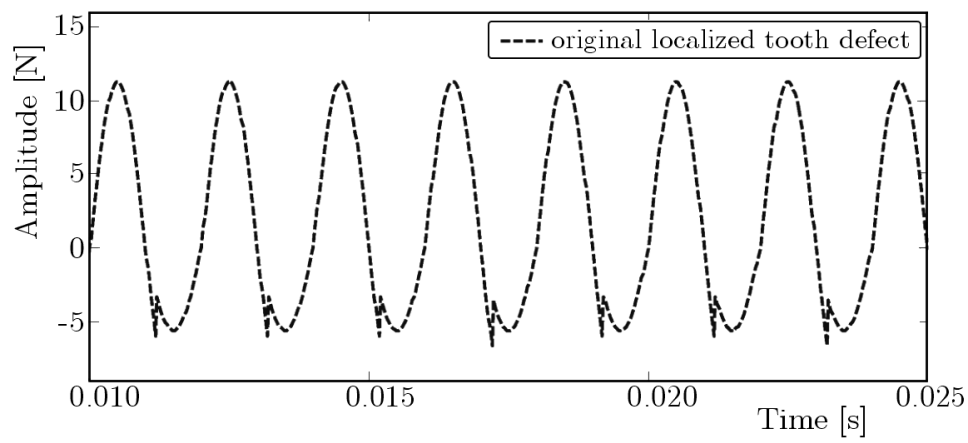

Fig. 3. Time-evolution of the localized tooth defect

In order to extract the original sources, the observed signals (accelerations) are determined at both the centre of the pinion and the centre of the gear and by taking into account the presence of the tooth failure and the eccentricity error. The rotational speed of the pinion is equal to $N=1500 \mathrm{tr} / \mathrm{min}$. The period is $T_{e}=0.002 \mathrm{~s}$ which corresponds to the gear mesh frequency $f_{e}=500 \mathrm{~Hz}$.

Then, these calculated dynamic reponses are used as inputs in the ICA separation algorithm to estimate the waveforms of the real internal defects in the spur gear as well as the excitation frequency and, finally, to identify the type of defect.

After determination of the observed signals, the ICA algorithm is applied to determine the estimated sources which correspond to the internal defects of the spur gear. The presence of the rotation frequency of the shaft in the spectrum of the estimated sources indicates clearly the position defect with the corresponding waveform. So, the eccentricity error can be identified. If only the gear mesh frequency and its harmonics are observed, we can stipulate that there is a tooth failure with the waveform and we can distinguish between the different tooth failures which may exist.

Figure 4 presents the evolution of the estimated source extracted by the ICA method from the observed signals versus time superimposed with the original source, which presents the eccentricity error. The estimated source has almost the same waveform and amplitude as the original source. The two sources have the same excitation frequency. 


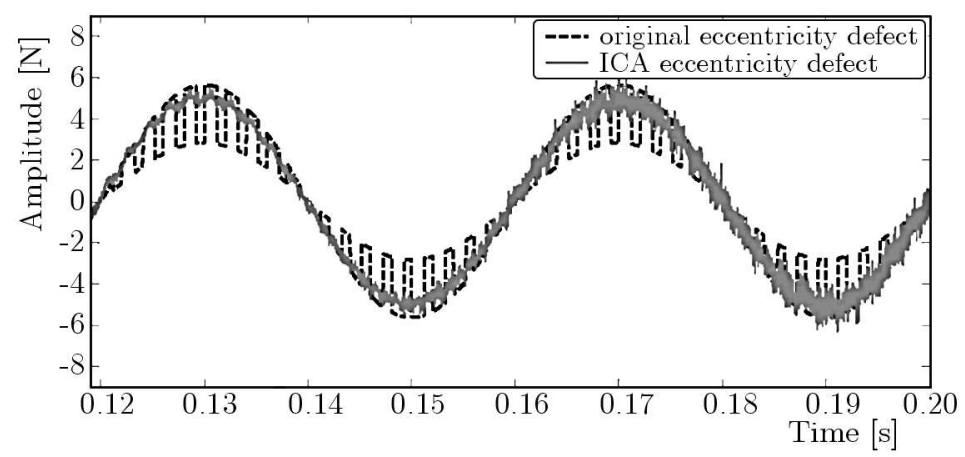

Fig. 4. Original and estimated time-evolution of the eccentricity defect

In Fig. 5, the spectrum of the estimated source corresponding to the eccentricity error is presented. We notice the presence of a peak at the rotation frequency of the shaft which is equal to $25 \mathrm{~Hz}$ and other peaks at the gear mesh frequency and its harmonics that correspond to the excitation frequency of the eccentricity error.

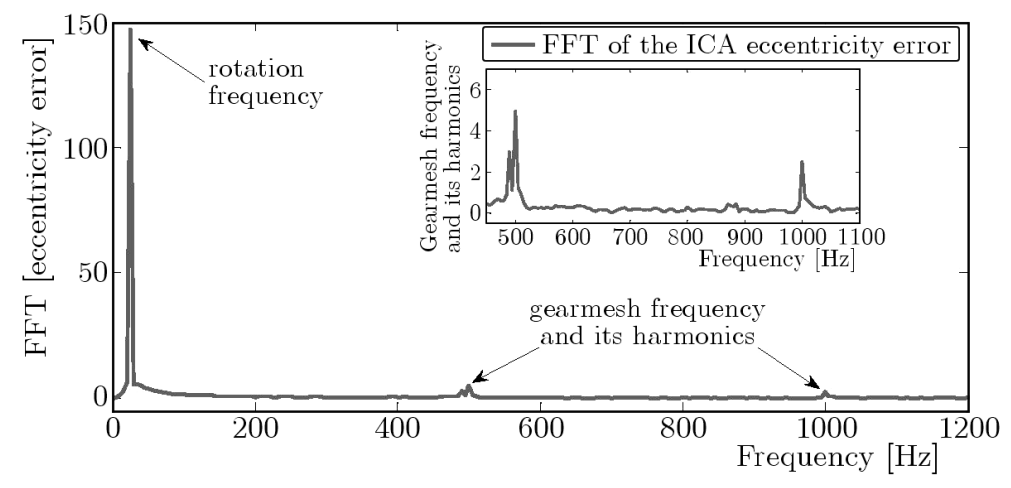

Fig. 5. Spectrum of the estimated signal of the eccentricity defect

Figure 6 shows the time-evolution of both original and estimated source corresponding to the tooth defect. So, one can clearly see the efficiency of the developed inverse method for reconstruction of the introduced internal defects. The amplitudes and the waveforms of the original and the estimated excitation source are almost identical.

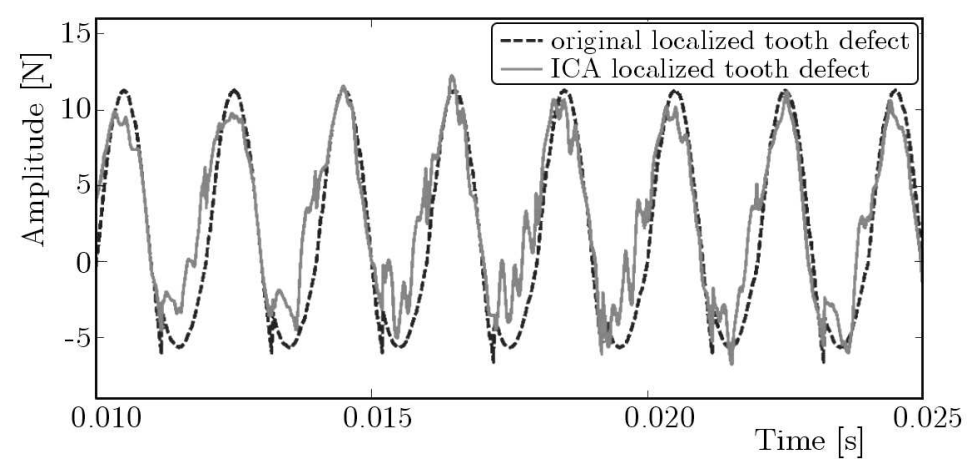

Fig. 6. Original and estimated time-evolution of the localized tooth defect

In Fig. 7, the spectrum of the second estimated source is presented. Here, we noticed the presence of three peaks corresponding to the gear mesh frequency and its harmonics. Hence, the excitation frequency of the tooth defect can be identified $\left(f_{e}=500 \mathrm{~Hz}\right)$. 


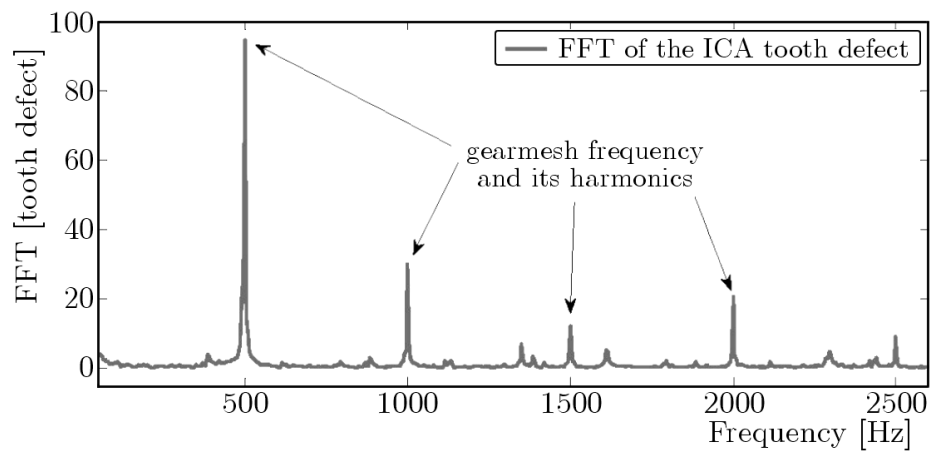

Fig. 7. Spectrum of the estimated signal of the second defect

From all the results presented above, we conclude that the estimated sources reconstructed by the developed inverse method(ICA algorithm) have the same waveforms and excitation frequencies as the internal defects considered present in the system. So, the ICA method gives good results for dynamic diagnosis of a one-stage spur gear and can be employed for other complicated mechanical systems.

\section{Conclusion}

In this paper, the ICA method has been presented and applied to a mechanical system defined bya one-stage spur gear in order to identify the eventual internal defects. Two different types of internal defects have been introduced in the equation of motion: the eccentricity error and tooth defect. Then, by using the dynamic response signals as inputs in the ICA algorithm, we have succeeded to recover the estimated sources which correspond to real internal sources introduced in the gear system. So, the usefulness of the proposed method is demonstrated.

\section{References}

1. Abbès M.S., Miladi Chaabane M., Akrout A., Fakhfakh T., Haddar M., 2011, Vibratory behavior of a double panel system by the operational modal analysis, International Journal of Modeling, Simulation and Scientific Computing, 2, 4, 459-479

2. Akrout A., Miladi Chaabane M., Hammami L., Haddar M., 2012, Edge stiffness effects on laminated double glazing system dynamical behaviour by the operational modal analysis, Journal of Mechanics of Materials and Structures, 7, 8/9, 837-859

3. Akrout A., Tounsi D., Taktak M., Abbes M.S., Haddar M., 2012, Estimation of dynamic system's excitation forces by the independent components analysis, International Journal of Applied Mechanics, 4, 3

4. Antoni J., Garibaldi L.,Marchesiello S., Sidahmed M., 2004, New separation techniques for output-only modal analysis, Shock and Vibration, 11, 227-242

5. Antoni J., 2005, Blind separation of vibration components: principles and demonstration, Mechanical Systems and Signal Processing, 19, 1166-1180

6. Antoni J., Braun S., 2005, Special issue on blind source separation: editorial, Mechanical Systems and Signal Processing, 19, 1163-1165

7. Chaari F., Baccar W., Abbès M.S., Haddar M., 2008,Effect of spelling or tooth breakage on gearmesh stiffness and dynamic response of a one-stage spur gear transmission, European Journal of Mechanics A/Solids, 27, 691-705 
8. Chanri F., Fakhfakh T., Haddar M., 2009, Analytical modelling of spur gear tooth crack and influence on gearmesh stiffness, European Journal of Mechanics A/Solids, 28, 461-468

9. Comon P., 1990, Separation of sources using higher-order cumulants, Actes Advanced Algorithms and Architectures for Signal Processing IV, San Diego, Californie, 1152, 170-181

10. Comon P., 1994, Independent component analysis: a new concept? Signal Processing, 36, 287-314

11. Gaeta M., Lacoume J.L., 1990, Maximum Likelihood Estimators Applied to the Non Gaussian Source Separation, Saint-Martin-d'Heres, France

12. Hidaka T., Terauchi Y., Dohi K., 1979, On the relation between the run out errors and the motion of the center of sun gear in a Stoeckicht planetary gear, Bulletin of JSME, 22, 167, 748-754

13. HYvärinen A., OJA E., 2000, Independent component analysis: algorithms and applications, Neural Networks, 13, 3/5, 411-430

14. Kerschen G., Poncelet F., Golinval J.C., 2007, Physical interpretation of independent component analysis in structural dynamics, Mechanical Systems and Signal Processing, 21, 1561-1575

15. Munro R., 1967, Gear transmission error, AGMA Paper, 239, 10

16. Poncelet F., Kerschen G., Golinval J.C., Verhelst D., 2007, Output-only modal analysis using blind source separation techniques, Mechanical Systems and Signal Processing, 21, 2335-2358

17. Servière C., FABry P., 2004, Blind source separation of noisy harmonic signals for rotating machine diagnosis, Journal of Sound and Vibration, 272, 317-339

18. Shalvi O., Weinstein E., 1990, New criteria for blind deconvolution of non-minimum phase systems (channels), IEEE Transactions Information Theory, 36, 2, 312-321

19. Taktak M., Tounsi D., Akrout A., Abbès M.S., Haddar M., 2012, One stage spur gear transmission crankcase diagnosis using the independent components method, International Journal of Vehicle Noise and Vibration, 8, 4, 387-400

20. Thi H.L.N., Jutten C., 1995, Blind source separation for convolutive mixtures, Signal Processing, 45, 209-229

21. Thirion-Moreau N., Moreau E., 2000, New criteria for blind signal separation, IEEE Workshop on Statistical Signal and Array Processing, 344-348

22. Tong L., Liu R.W., Soon V.C., Huang Y.F., 1991, Indeterminacy and identifiability of blind identification, IEEE Transactions on Circuits and Systems, 38, 499-509

23. Wajag P., Kahraman A., 2002, Influence of tooth profile modification on helical gear durability, ASME Transactions, Journal of Mechanical Design, 124, 501-510

24. Wiggins R.A., 1978, Minimum entropy deconvolution, Geoexploration, 16, 21-35

25. Zang C., Friswel M.I., Imregun M., 2004, Structural damage detection using independent component analysis, Structural Health Monitoring, 3, 1, 69-83

26. Zarzoso V., Comon P., 2010, Robust independent component analysis by iterative maximization of the Kurtosis contrast with algebraic optimal step size, IEEE Transactions on Neural Networks, 21, 2 